\title{
COMMUNICATIONS
}

\section{Characterization of nanoblisters on HOPG surface}

\author{
Dinara Sobola*, Nikola Papež ${ }^{*}$, Rashid Dallaev*, \\ Shikhgasan Ramazanov $^{* *}$, Dušan Hemzal ${ }^{* * *}$, Vladimír Holcman*
}

\begin{abstract}
We report on influence of the surface functionalization on the properties of highly oriented pyrolytic graphite. The samples were processed in nitric acid and characterized by XPS, Raman and EDX spectroscopies, AFM, SEM and optical microscopy. It is shown that interaction of nitric acid with the surface of HOPG leads to two types of reactions: oxidation of the graphite and intercalation of the nitrate ions at the blistered areas.

K e y w or d s: graphite, nitric acid, surface, delamination, topography
\end{abstract}

\section{Introduction}

Under normal conditions, graphite is the most stable allotropic form of carbon. Variation of its physical and chemical properties define the application range of graphite from pencil leads to cutting-edge calibration etalons in scientific applications. Thermal characteristics of graphite make it interesting for aerospace applications [1]. Intercalation of compounds can further spread properties of graphite and increase is application potential.

Both HOPG (highly oriented pyrolytic graphite) and HAPG (highly annealed pyrolytic graphite) exhibit pronounced layered structure. HOPG consists of hexagonal carbon planes situated in ABAB positions. Due to Van der Waals bonding between the planes this material is utilized for study and preparation of graphene structures. The interplanar distance between layers can be changed by intercalation of metals.

Graphene nanoblisters and nanobubbles are partially delaminated areas of one or several graphite layers. Both can be prepared on graphite itself or on interfaces of graphite with substrates from other materials. The nanobubbles are of interest because they can trap molecules from gases [2]. There are several methods of graphite nanobubbles formation on HOPG surface: pure chemical, electrochemical, plasma processing, etc [3]. Formation of blisters consists of penetration of the reagent through defects into near-surface layers and oxidation of graphite. In result, formation and dissociation of carbonic acid leads to blistering.

Braking of interplanar bonds at HOPG near-surface area and appearance of round-shaped blisters were reported before [4]-[6]. Our study contributes to this field by confirmation of the theoretical work of Tang and Cao
[7] about stronger adsobtion of $\mathrm{NO}_{3}$ in comparison to other nitrogen oxides. The $\mathrm{NO}_{3}$ anion could be detected by Raman spectrsocopy at the blistered areas. We also demonstrate by XPS and Raman spectroscopies presence of $\mathrm{NH}_{2}$ group whish is important for surface functionalization for biomedical application [8].

Appearance of blisters, delamination and "bubbles" could also occur during films deposition using carbon materials as substrates. Since control of the surface condition of the substrate is important part of deposition technologies $[4,5]$, modified carbon surfaces are thus of interest also for growth control during deposition of thin films [11].

\section{Experiment}

HOPG samples of ZYB quality (purchased from TipsNano OÜ) were exfoliated by tape and exposed to $65 \%$ nitric acid $\left(\mathrm{HNO}_{3}\right)$ under ambient conditions for 8 minutes. After the exposition, the samples were washed using demineralized water. Optical imaging of the samples before treatment and after treatment is shown in Fig. 1. The position of AFM probe is shown in Fig. 1(a).

The obtained surfaces were studied using atomic force microscopy (AFM) in semi-contact mode in order to not damage the surface features [7, 8]. The blistered areas tend to form "bubbles" on the surface with height up to $1 \mu \mathrm{m}$ (see Fig. 2). It was suggested by Burgess at al. [14] that the blistering occurs due to functionalization of the surface defects by oxygen-containing bonds [15].

The blistered areas were also observed by SEM (scanning electron microscopy): maximum diameter of the nanobubbles was up to $3 \mu \mathrm{m}$ (see Fig, 3 ).

*Department of Physics, Faculty of Electrical Engineering and Communication, Brno University of Technology, Technick 2848/8, 616 00 Brno, Czech Republic, nikola.papez@vutbr.cz, **Faculty of Physics, Dagestan State University, 367015 Makhachkala, st. M. Gadjieva 43-a, Dagestan Republic, Russia, ***Department of Condensed Matter Physics, Faculty of Science, Masaryk University, Kotlářská 267/2, 61137 Brno, Czech Republic

DOI: 10.2478 /jee-2019-0055, Print (till 2015) ISSN 1335-3632, On-line ISSN 1339-309X 

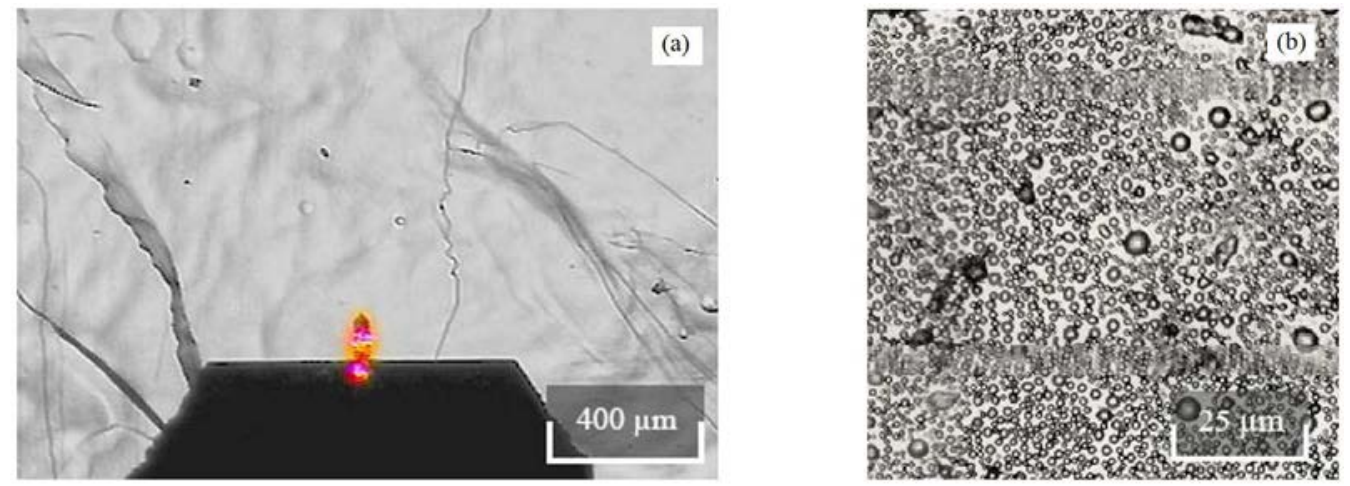

Fig. 1. Optical images: HOPG (ZYB) surface: (a) - before and, (b) - after 8 minutes of processing by $\mathrm{HNO}_{3}$
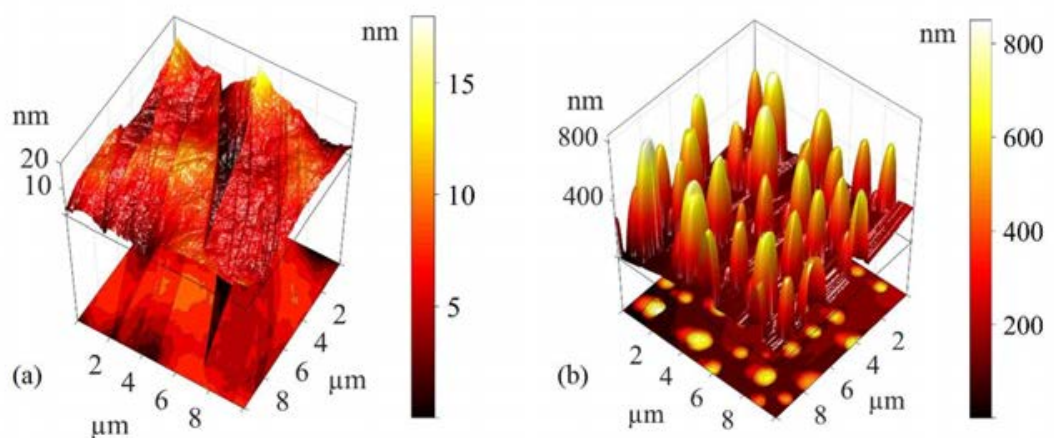

Fig. 2. AFM images: HOPG (ZYB) surface: (a) - before, and (b) - after 8 minutes processing in $\mathrm{HNO}_{3}$.
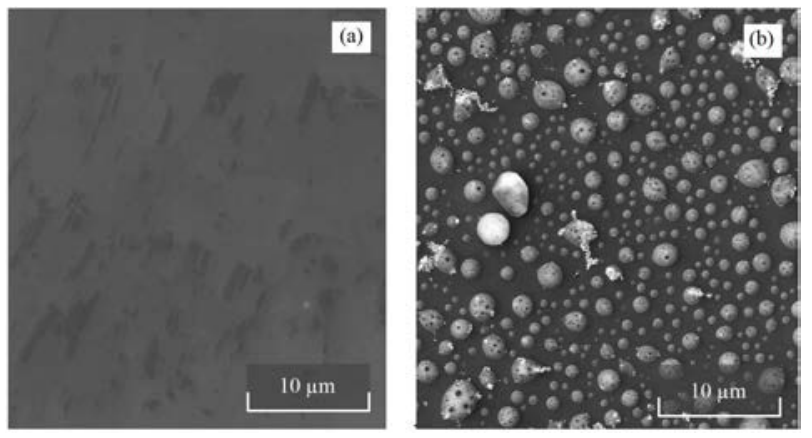

Fig. 3. SEM images: HOPG (ZYB) surface: (a) - before, and (b) - after 8 minutes of processing by $\mathrm{HNO}_{3}$

Concerning chemical analysis, XPS showed presence of several peaks after treatment of HOPG surface with nitric acid (see Fig. 4). The N1s nitrogen peak occurring at $\sim 400 \mathrm{eV}$ is usually assigned to $\mathrm{C}-\mathrm{NH}_{2}$ bond and its appearance corresponds with theory [14]. However, only 2 minutes of etching by $5 \mathrm{keV}$ argon ions is sufficient to almost completely remove N1s peak, Fig. 4(b). It happens because of preferential etching of nitrogen which occurs by Ar irradiation. The other peak that emerged after $\mathrm{HNO}_{3}$ treatment is the oxygen peak O1s at $532.4 \mathrm{eV}$ corresponding to the $\mathrm{C}-\mathrm{O}$ bond. To remove the $\mathrm{O} 1 \mathrm{~s}$ peak entirely, much longer Ar exposition would be required: even after 20 minutes of etching remnants of this peak persist, Fig. 4(c). Ion beam etching was performed in situ in the XPS chamber. The samples were not exposed to air in order to avoid influence of atmospheric oxygen to the surface condition.

Additionally, EDS (Energy Dispersive X-Ray Spectroscopy) was used for determination of oxygen abundance in the near-surface area. Although EDS did not prove the presence of nitrogen (which was observed by XPS due to its higher sensitivity to surface condition), oxygen content in the measured samples increased from 0.59 up to 1.02 atomic \% after treatment.

The amount of oxygen increased twice after treatment, which confirms redox reaction between HOPG and $\mathrm{HNO}_{3}$. First, surfactant elements from the acid create chemical bonds with graphite surface during reactions:

$$
\mathrm{C}+4 \mathrm{HNO}_{3} \longrightarrow \mathrm{H}_{2} \mathrm{CO}_{3}+4 \mathrm{NO}_{2} \text {. }
$$

Subsequently, the carbonic acid instantly decomposes into carbon dioxide and water:

$$
\mathrm{C}+4 \mathrm{HNO}_{3} \longrightarrow \mathrm{CO}_{2}+2 \mathrm{H}_{2} \mathrm{O}+4 \mathrm{NO}_{2} \text {. }
$$

The produced stress occurs between the reacted nearsurface area and the rest of the sample. The delamination of the layers and formation of blisters occurs where Van der Waals bonds are broken. Finally, Raman spectroscopy is widely used for characterization of carbon materials. We used Renishaw InVia spectrometer with laser power of $1 \mathrm{~mW}$ and wavelength $532 \mathrm{~nm}$ [16]. The observed Raman peaks (see Fig. 5) correspond to graphite intercalation 

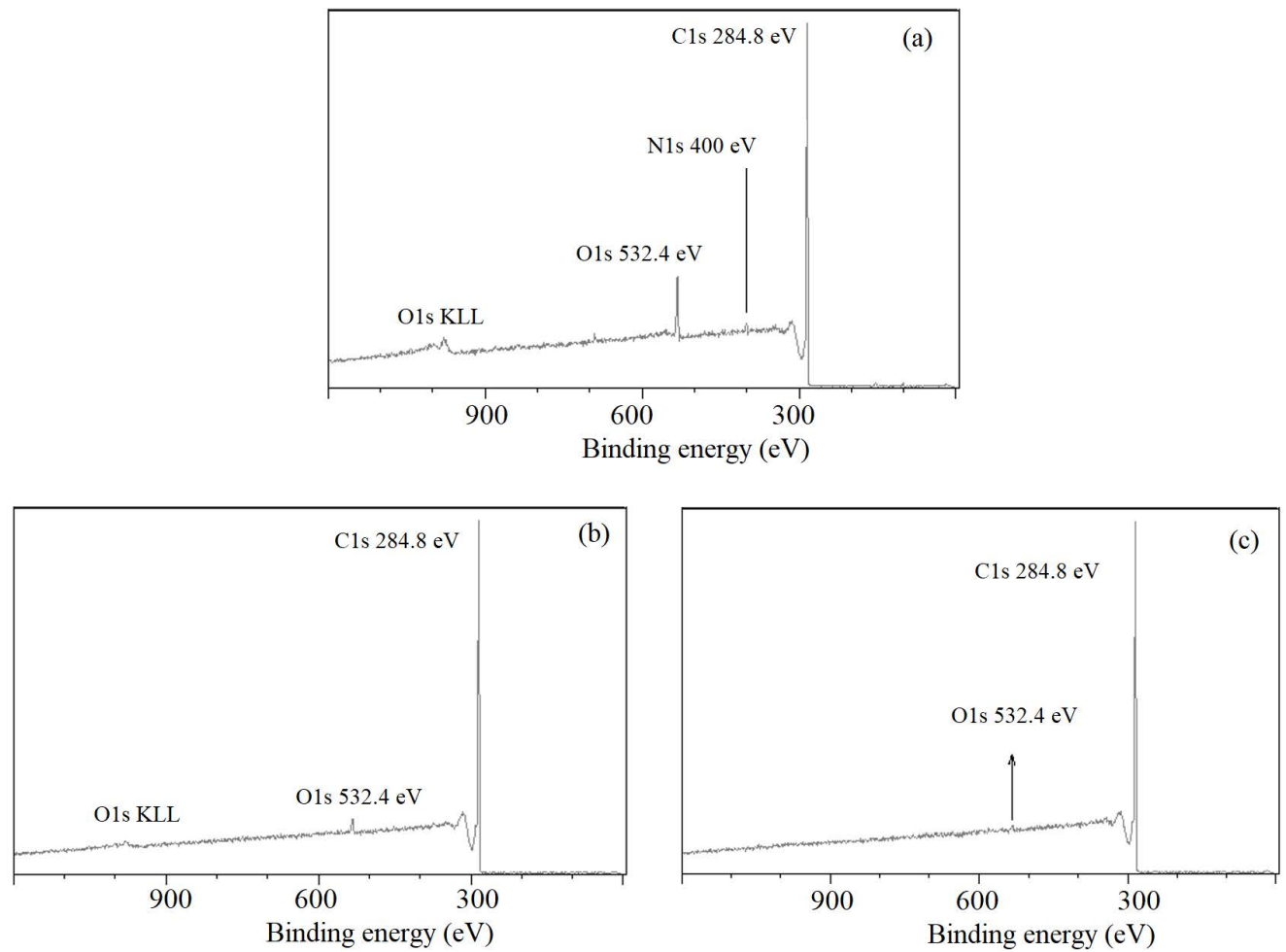

Fig. 4. Wide XPS spectra of $\mathrm{HOPG}$ treated with $\mathrm{HNO}_{3}$ during 8 minutes: (a) - before Ar etching, (b) - 2 min Ar etching, and (c) - 20 min Ar etching

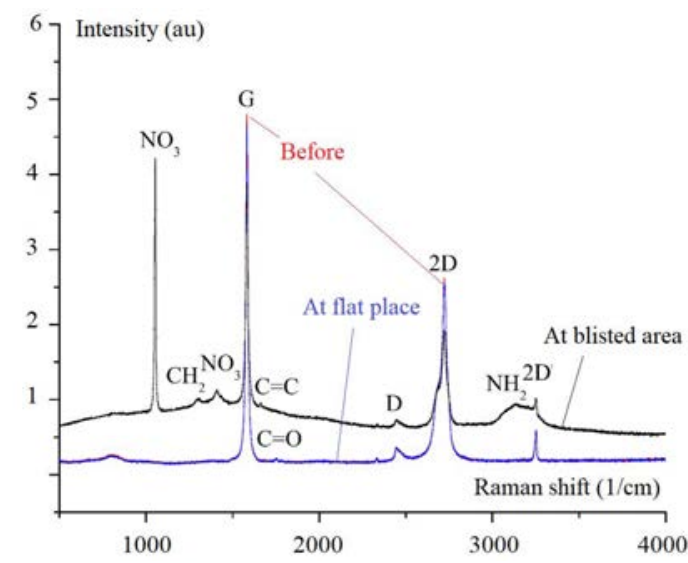

Fig. 5. Raman spectra: HOPG (ZYB) surface before and after 8 minutes of processing by $\mathrm{HNO}_{3}$

compounds. According to Dunaev and Shapoev [17] the intercalation process could be presented as:

$$
\begin{aligned}
& 2 \mathrm{HNO}_{3} \text { adsorbed } \longrightarrow \mathrm{H}_{2} \mathrm{O}+\mathrm{NO}_{3}^{-}+\mathrm{NO}_{2}^{+} \text {adsorbed } \\
& \mathrm{NO}_{2}^{+} \text {adsorbed }+C \longrightarrow \mathrm{NO}_{2} \text { adsorbed }+C^{+} \\
& \mathrm{NO}_{2} \text { adsorbed } \longrightarrow \mathrm{NO}_{2} \text { gas } \\
& \mathrm{nC}^{+}+\mathrm{NO}_{3}^{-}+\mathrm{xHNO}_{3} \longrightarrow \mathrm{C}_{n}^{+} \mathrm{NO}_{3}^{-} \mathrm{xHNO}_{3}
\end{aligned}
$$

The main differences in comparison to pristine $\mathrm{HOPG}$ are in spectra from the blister areas. The common areas are observed at $1582 \mathrm{~cm}^{-1}$ and $2721 \mathrm{~cm}^{-1}$ which are positions of $\mathrm{G}$ and $2 \mathrm{D}$ peaks of $\mathrm{HOPG}$ [18]. Doublet of $2450 \mathrm{~cm}^{-1}$ and $3248 \mathrm{~cm}^{-1}$ is assigned to D and 2D modes of HOPG, correspondingly [19]. The blister areas show, however, additional features:
- peak at $1053 \mathrm{~cm}^{-1}$ refers to macro-cation $\mathrm{NO}_{3}$ trapped between the graphite layers and the rest of nondissociated $\mathrm{HNO}_{3}$ [20],

- peak at $1300 \mathrm{~cm}^{-1}$ is connected with transverse vibrations of $\mathrm{CH}_{2}$ group [21],

- appearance of the $1409 \mathrm{~cm}^{-1}$ peak can be attributed to incorporated nitrate anion [22],

- features of $\mathrm{C}=\mathrm{C}$ and $\mathrm{C}=\mathrm{O}$ stretching modes are noted by $1660 \mathrm{~cm}^{-1}$ and $1748 \mathrm{~cm}^{-1}$ peaks, correspondingly [23],

- peak at $3130 \mathrm{~cm}^{-1}$ occurs due to $\mathrm{NH}_{2}$ groups [24].

Thus, the Raman measurement confirms presence of bonds which result from intercalation and oxidation reactions [25]. In order to check the inner structure of the surface features which occurs after the acid treatment the depth-scan Raman measurement was done. The largest blister on the surface was funded by optical microscope for this aim, Fig. 6(a). Contrasted images for $\mathrm{NO}_{3}$ peak and G-peak at the surface, in middle( and at the bottom are investigated (Fig. 6(b)-(d). The results indicate presence of $\mathrm{NO}_{3}^{-}$ions and intensive G-peak on the surface. G-peak decreases during scanning inside the blisters and growths with approaching to the HOPG bulk. The content of nitrogen oxide is higher in the middle of the blister. Peak ratio demonstrates presence of several carbon layers on the surface of the delaminated area and higher amount of the nitrogen compounds $\left(\mathrm{NO}_{3}\right.$ and $\left.\mathrm{NH}_{2}\right)$ below them. 
(a)
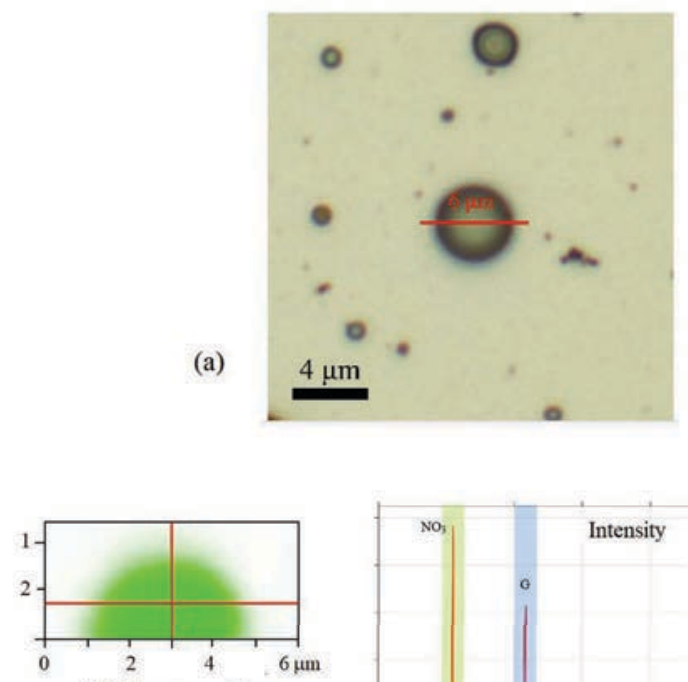

m

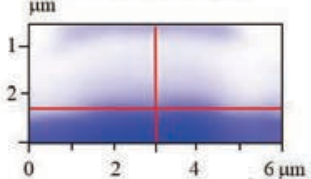

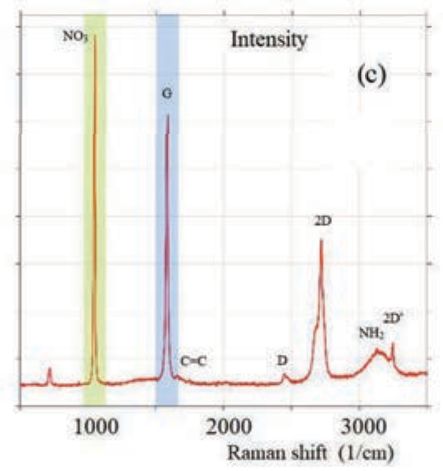
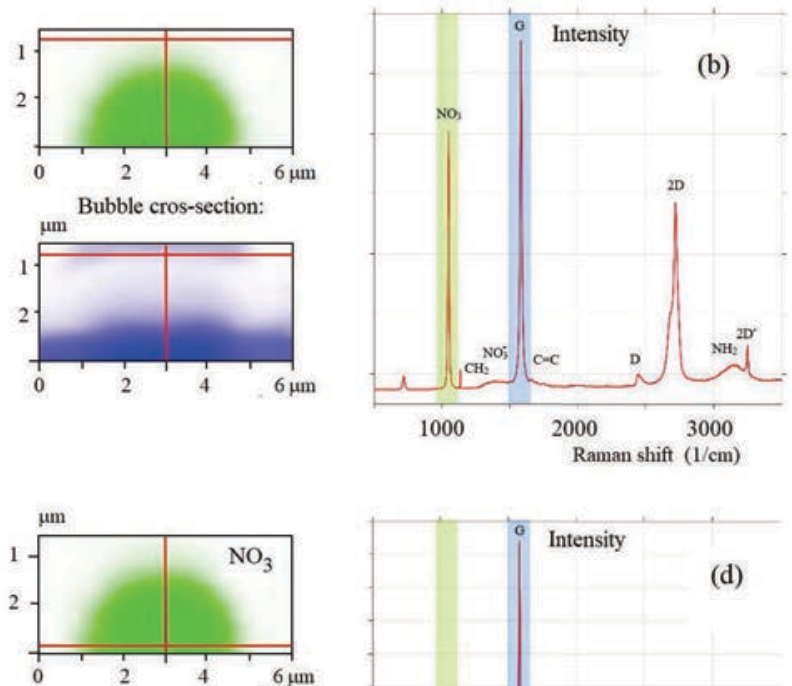

Bubble cros-section:
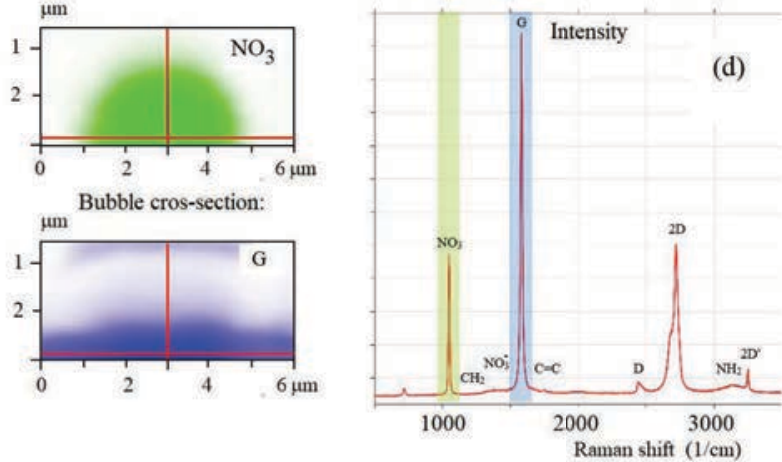

Fig. 6. Raman spectra of the blistered area in depth: (a) - optical top view of the blister for Raman depth-scan, (b) - blister cross-section, top view, (c) - bBlister cross-section, middle view, and (d) - blister cross-section, bottom view

\section{Conclusion}

Treatment of the surface by $\mathrm{HNO}_{3}$ created blistered areas. AFM, SEM and optical images demonstrate formation of nanoblisters with different size but similar shape. According to EDS, there are changes in oxygen abundance in the near-surface area after processing by $\mathrm{HNO}_{3}$.

In summary, nitric acid attacks graphite as oxidant by taking forth an electron (three electrons participate in plain covalent bonding). It is expected that $\mathrm{CO}_{2}, \mathrm{NO}_{2}$ and water occur in result of reaction between $\mathrm{HNO}_{3}$ and graphite. Intercalated elements consist of products of redox reactions between graphite and $\mathrm{HNO}_{3}$. We observed the presence of electron acceptor $\mathrm{NO}_{3}^{-}$at the blistered areas. XPS and Raman data confirmed surface functionalization by $\mathrm{NH}_{2}$ group.

All these defects influence physical and chemical properties of the surface and also affect the performance of the material in its potential applications. In particular, formation of blisters should be considered when HOPG is used as a substrate for epitaxy and heterostructures preparation.

\section{Acknowledgements}

Research described in the paper was financially supported by the Ministry of Education, Youth and Sports of the Czech Republic under the project CEITEC 2020 (LQ1601), by the National Sustainability Program under grant LO1401, and by Internal Grant Agency of Brno
University of Technology, Grant No. FEKT-S-17-4626. For the research, infrastructure of the SIX Center was used. Part of the work was carried out with the support of CEITEC Nano Research Infrastructure (MEYS CR, 2016-2019).

\section{REFERENCES}

[1] E. Ancona and R. Y. Kezerashvili, "Temperature restrictions for materials used in aerospace industry for the near-Sun orbits", Acta Astronaut., 2017.

[2] E. Khestanova, F. Guinea, L. Fumagalli, A. K. Geim, and I. V. Grigorieva, "Universal shape and pressure inside bubbles appearing in van der Waals heterostructures", Nat. Commun., 2016.

[3] L. Liu, M. Qing, Y. Wang, and S. Chen, "Defects in Graphene: Generation, Healing, and Their Effects on the Properties of Graphene: A Review", J. Mater. Sci. Technol., 2015.

[4] S. Yang, P. Tsai, E. S. Kooij, A. Prosperetti, H. J. W. Zandvliet, and D. Lohse, "Electrolytically generated nanobubbles on highly orientated pyrolytic graphite surfaces", Langmuir, 2009.

[5] E. Bouleghlimat, P. R. Davies, R. J. Davies, R. Howarth, J. Kulhavy, and D. J. Morgan, "The effect of acid treatment on the surface chemistry and topography of graphite", Carbon N. Y., 2013 .

[6] O. V. Sinitsyna, G. B. Meshkov, A. V. Grigorieva, A. A. Antonov, I. G. Grigorieva, and I. V. Yaminsky, "Blister formation during graphite surface oxidation by Hummers method", Beilstein J. Nanotechnol., 2018.

[7] S. Tang and Z. Cao, "Adsorption of nitrogen oxides on graphene and graphene oxides: Insights from density functional calculations", J. Chem. Phys., 2011. 
[8] H. J. Kim et al., "Synthesis and characteristics of NH 2-functionalized polymer films to align and immobilize DNA molecules", Nanoscale Res. Lett., 2012.

[9] M. M. Koc, M. J. McNally, K. von Haeften, and M. J. Watkins, "AFM induced self-assembling and self-healing mechanism of silicon oxide nanoparticle linear array domains templated on Moir superlattice patterns on HOPG", Surf. Sci., 2019.

[10] M. Kettner, C. Stumm, M. Schwarz, C. Schuschke, and J. Libuda, "Pd model catalysts on clean and modified HOPG: Growth, adsorption properties, and stability", Surf. Sci., 2019.

[11] R. Shikhgasan, T. Stefan, S. Dinara, S. Sebastian, and R. Guseyn, "Epitaxy of silicon carbide on silicon: Micromorphological analysis of growth surface evolution", Superlattices Microstruct., vol. 86, pp. 395-402, 2015.

[12] D. Sobola, S. Talu, S. Solaymani, and L. Grmela, "Influence of scanning rate on quality of AFM image: Study of surface statistical metrics", Microsc. Res. Tech., vol. 80, no. 12, 2017.

[13] S. Talu, D. Sobola, and N. Papež, "Analysis and Recommendations for Education Process of Experts in the Field of Scanning Probe Microscopy", DEStech Trans. Soc. Sci. Educ. Hum. Sci., vol. 0, no. aetms, 2017.

[14] R. Burgess et al., "The functionalisation of graphite surfaces with nitric acid: Identification of functional groups and their effects on gold deposition", J. Catal., 2015.

[15] S. Talu, D. Sobola, S. Solaymani, R. Dallaev, and J. Brüstlová, "Scale-depended Choice of Scanning Rate for AFM Measurements", DEStech Trans. Comput. Sci. Eng., no. cnai, 2018.

[16] N. Papež et al., "Degradation analysis of GaAs solar cells at thermal stress", Appl. Surf. Sci., 2018.

[17] A. S. A. Dunaev, "Prolific Family of Carbon Materials", Online: http://www.nanometer.ru.
[18] P. H. Tan, Y. M. Deng, Q. Zhao, and W. C. Cheng, "The intrinsic temperature effect of the Raman spectra of graphite", Appl. Phys. Lett., 1999.

[19] P. H. Tan, Y. M. Deng, and Q. Zhao, "Temperature-dependent Raman spectra and anomalous Raman phenomenon of highly oriented pyrolytic graphite", Phys. Rev. B - Condens. Matter Mater. Phys., 1998.

[20] R. L. F. J. Theo Kloprogge, D. Wharton, L. Hickel, "Infrared and Raman study of interlayer anions CO3 2- , NO3 - , SO4 2and $\mathrm{ClO} 4$ - in Mg/Al- hydrotalcite", Am. Mineral., 2002.

21] R. W. C. G. Wood, "Raman Spectra of a Series of Normal Alcohols and Other Compounds", vol. 42, no. 1, pp. 386-393, 1932 .

[22] V. Rives, "Layered Double Hydroxides: Present and Future", 2011.

[23] P. Meksiarun, N. Spegazzini, H. Matsui, K. Nakajima, Y. Matsuda, and H. Satoa, "In vivo study of lipid accumulation in the microalgae marine diatom Thalassiosira pseudonana using Raman spectroscopy", Appl. Spectrosc., 2015.

[24] A. L. M. B. De Carvalho, S. M. Fiuza, J. Tomkinson, L. A. E. B. De Carvalho, and M. P. M. Marques, "Pt(II) complexes with linear diamines-Part I: Vibrational study of Pt-diaminopropane", Spectrosc. (New York), 2012.

[25] N. Papež et al., "Surface morphology after reactive ion etching of silicon and gallium arsenide based solar cells", J. Phys. Conf. Ser., vol. 1124, pp. 041015, 2018.

Received 19 March 2019 